\title{
Casimir-Polder interaction between an atom and a cylinder with application to nanosystems
}

\author{
G L Klimchitskaya ${ }^{1}$, E V Blagov ${ }^{2}$ and V M Mostepanenko ${ }^{2}$ \\ ${ }^{1}$ North-West Technical University, Millionnaya St. 5, St.Petersburg, Russia \\ ${ }^{2}$ Noncommercial Partnership "Scientific Instruments", Moscow, Russia.
}

\begin{abstract}
Recently the Lifshitz theory of dispersion forces was extended for the case of an atom (molecule) interacting with a plane surface of a uniaxial crystal or with a long solid cylinder or cylindrical shell made of isotropic material or uniaxial crystal. The obtained results are applicable to nanosystems. In particular, we investigate the Casimir-Polder interaction between hydrogen atoms (molecules) and multi-wall carbon nanotubes. It is demonstrated that the hydrogen atoms located inside multiwall carbon nanotubes have a lower free energy compared to those located outside. We also perform comparison studies of the interaction of hydrogen atoms between themselves and with multi-wall carbon nanotube. The obtained results are important for the problem of hydrogen storage.
\end{abstract}

PACS numbers: 73.22.-f, 12.20.Ds, 34.50.Dy, 34.20.Cf

In the last few years, carbon nanotubes and other nanostructures attracted much attention in connection with the problem of hydrogen storage (see Refs. [1, 2] for a review). Many important properties of carbon nanotubes are determined by the van der Waals and Casimir-Polder interactions. In particular, the Casimir-Polder forces acting between hydrogen atoms or molecules and carbon nanotubes play the main role in absorption phenomena. However, for a long time they were practically unexplored. Recently, modern methods using density functional theory, quantum molecular dynamics and Monte Carlo simulations were applied to investigate the interaction of hydrogen atoms and molecules with single-wall nanotubes [3-6] and fullerenes [7, 8]. Multi-wall carbon nanotubes with sufficiently many layers can be modeled by a graphite cylindrical shell of some length $L$, external radius $R \ll L$, and thickness $d<R$. In this case the crystal optical axis $z$ is perpendicular to the surface of the shell formed by the hexagonal layers of a graphite lattice and the material of the shell is described by the dielectric permittivities $\varepsilon_{x}(\omega)=\varepsilon_{y}(\omega)$ and $\varepsilon_{z}(\omega)$.

In Ref. 9] the Lifshitz formula for the Casimir-Polder interaction between an atom and a semispace made of isotropic material [10, 11] was generalized for the cases when a semispace is replaced by a plate of finite thickness made of a uniaxial crystal or by 
a cylindrical shell made of the same material. In this paper we apply the obtained Lifshitz-type formulas to describe the Casimir-Polder interaction of hydrogen atoms and molecules with multi-wall carbon nanotubes. In the case of atom or molecule with a dynamic polarizability $\alpha(\omega)$ at separation $a$ from a plane surface of a plate made of a uniaxial crystal at temperature $T$ at thermal equilibrium, the free energy of the Casimir-Polder interaction is given by [9]

$$
\begin{aligned}
& \mathcal{F}^{p}(a, T)=-\frac{k_{B} T}{8 a^{3}}\left\{2 \alpha(0)+\sum_{l=1}^{\infty} \alpha\left(i \zeta_{l} \omega_{c}\right)\right. \\
& \left.\times \int_{\zeta_{l}}^{\infty} d y e^{-y}\left[\left(2 y^{2}-\zeta_{l}^{2}\right) r_{\|}\left(\zeta_{l}, y\right)+\zeta_{l}^{2} r_{\perp}\left(\zeta_{l}, y\right)\right]\right\} .
\end{aligned}
$$

Here the crystal optical axis $z$ is perpendicular to the plane of plates $(x, y), k_{B}$ is the Boltzmann constant, $\zeta_{l}=4 \pi k_{B} l T a /(\hbar c)$ are the dimensionless Matsubara frequencies, $\omega_{c}=c /(2 a)$ is the characteristic frequency, and the reflection coefficients are given by

$$
\begin{aligned}
& r_{\|}\left(\zeta_{l}, y\right)=\frac{\varepsilon_{x l} \varepsilon_{z l} y^{2}-f_{z}^{2}\left(y, \zeta_{l}\right)}{\varepsilon_{x l} \varepsilon_{z l} y^{2}+f_{z}^{2}\left(y, \zeta_{l}\right)+2 \sqrt{\varepsilon_{x l} \varepsilon_{z l}} y f_{z}\left(y, \zeta_{l}\right) \operatorname{coth}\left[f_{z}\left(y, \zeta_{l}\right) d /(2 a)\right]}, \\
& r_{\perp}\left(\zeta_{l}, y\right)=\frac{f_{x}^{2}\left(y, \zeta_{l}\right)-y^{2}}{y^{2}+f_{x}^{2}\left(y, \zeta_{l}\right)+2 y f_{x}\left(y, \zeta_{l}\right) \operatorname{coth}\left[f_{x}\left(y, \zeta_{l}\right) d /(2 a)\right]},
\end{aligned}
$$

where

$$
\begin{aligned}
& f_{z}^{2}\left(y, \zeta_{l}\right)=y^{2}+\zeta_{l}^{2}\left(\varepsilon_{z l}-1\right), \quad f_{x}^{2}\left(y, \zeta_{l}\right)=y^{2}+\zeta_{l}^{2}\left(\varepsilon_{x l}-1\right), \\
& \varepsilon_{z l} \equiv \varepsilon_{z}\left(i \zeta_{l} \omega_{c}\right), \quad \varepsilon_{x l} \equiv \varepsilon_{x}\left(i \zeta_{l} \omega_{c}\right) .
\end{aligned}
$$

In the limiting case of $d \rightarrow \infty$ Eqs. (11)-(3) give us the free energy of atom-semispace interaction $\mathcal{F}^{s}(a, T)$.

The free energy of the interaction between an atom and a cylinder shell is [9]

$$
\begin{aligned}
& \mathcal{F}^{c}(a, T)=-\frac{k_{B} T}{8 a^{3}} \sqrt{\frac{R}{R+a}}\left\{\frac{4 R+3 a}{2(R+a)} \alpha(0)+\sum_{l=1}^{\infty} \alpha\left(i \zeta_{l} \omega_{c}\right)\right. \\
& \left.\times \int_{\zeta_{l}}^{\infty} d y y e^{-y}\left[y-\frac{a}{2(R+a)}\right]\left[\left(2-\frac{\zeta_{l}^{2}}{y^{2}}\right) r_{\|}\left(\zeta_{l}, y\right)+\frac{\zeta_{l}^{2}}{y^{2}} r_{\perp}\left(\zeta_{l}, y\right)\right]\right\} .
\end{aligned}
$$

This formula was obtained using the proximity force theorem and the approximate expression for the interaction energy of two concentric cylinders [12. It is practically exact at $a \ll R$ and has an error of about $1 \%$ at all $a \leq R / 2$.

We have computed the free energy of the Casimir-Polder interaction between a hydrogen atom or molecule and a graphite semispace or multi-wall carbon nanotube using Eqs. (11) and (4), respectively. The dynamic polarizabilities of a hydrogen atom and molecule were represented in the framework of a single oscillator model [13]

$$
\alpha\left(i \xi_{l}\right)=\alpha_{a, m}\left(i \xi_{l}\right)=\frac{g_{a, m}}{\omega_{a, m}^{2}+\xi_{l}^{2}}
$$

where the parameters $g_{a, m}=\alpha_{a, m}(0) \omega_{a, m}^{2}$ are expressed through the static polarizabilities of an atom and a molecule $\alpha_{a}(0)=4.50$ a.u., $\alpha_{m}(0)=5.439$ a.u. and 


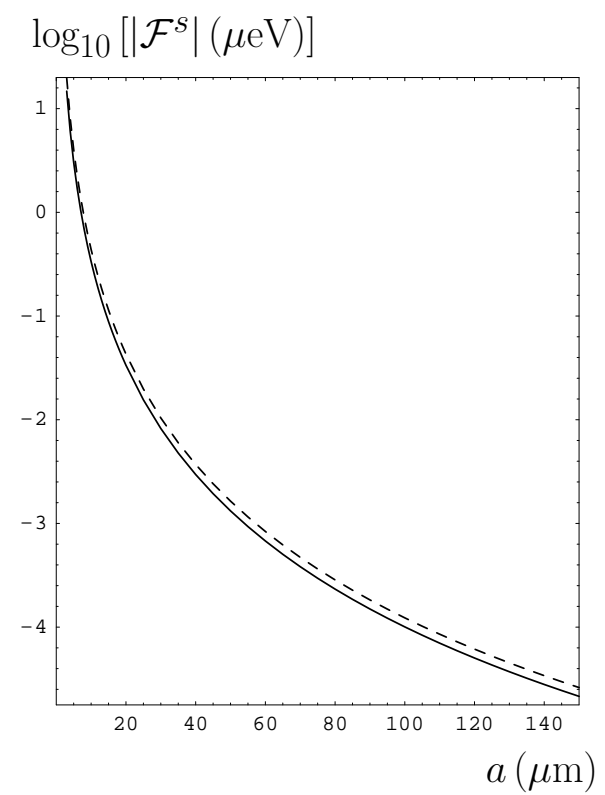

Figure 1. Magnitude of the free energy of a hydrogen atom (solid line) and molecule (dashed line) in a logarithmic scale as a function of separation from a graphite semispace.

the characteristic energies $\omega_{a}=11.65 \mathrm{eV}$ and $\omega_{m}=14.09 \mathrm{eV}$, respectively (recall that 1 atomic unit of polarizability is equal to $1.482 \times 10^{-31} \mathrm{~m}^{3}$ ). With a precision of better than $1 \%$ the single-oscillator model leads to the same results for the free energy as the highly accurate 10-oscillator model [14. The dielectric permittivities of graphite $\varepsilon_{x, z}\left(i \zeta_{l} \omega_{c}\right)$ were found by means of dispersion relation using the tabulated optical data for its complex index of refraction [15. In Fig. 1 we present the computation results for the magnitudes of the Casimir-Polder free energy at $T=300 \mathrm{~K}$ as a function of separation when hydrogen atom (solid line) or molecule (dashed line) interact with a graphite semispace. As is seen from Fig. 1, the magnitude of the free energy for the hydrogen molecule is larger than for the atom by $33 \%, 26 \%$, and $21.6 \%$ at separations $a=3 \mathrm{~nm}, 30 \mathrm{~nm}$, and $150 \mathrm{~nm}$, respectively.

In Fig. 2(a) we compare the free energies of hydrogen atoms located outside and inside a multi-wall carbon nanotube. The free energy of an atom outside a nanotube, $\mathcal{F}^{c, \text { ext }}=\mathcal{F}^{c}(a, T)$, was computed by Eq. (4) , and inside a nanotube, $\mathcal{F}^{c, \text { int }}$, was obtained by the method of additive summation of the interatomic van der Waals potentials [16, 17]. In Fig. 2(a) the difference of these energies is presented as a function of nanotube thickness in the case that both atoms are located at a separation $a=3 \mathrm{~nm}$ from the external and internal surfaces of a nanotube, respectively. Nanotubes with the fixed internal radius, $R_{0}=10 \mathrm{~nm}$, are shown by the solid line, whereas nanotubes with the fixed external radius, $R=50 \mathrm{~nm}$, are represented by the dashed line. As is seen from Fig. 2(a), $\mathcal{F}^{c, e x t}-\mathcal{F}^{c, i n t}>0$, i.e., the position of an atom inside a nanotube is 


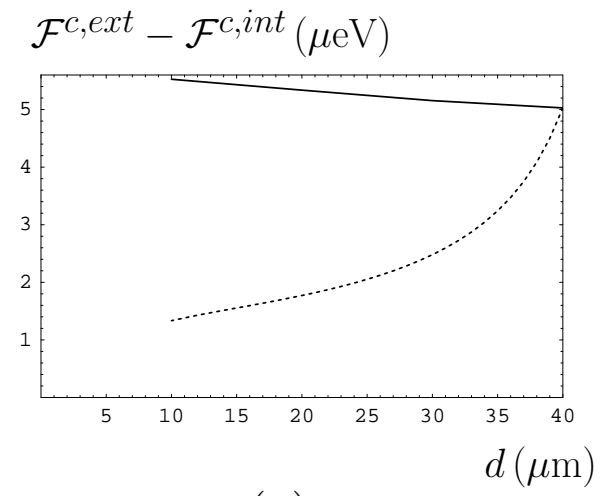

(a)

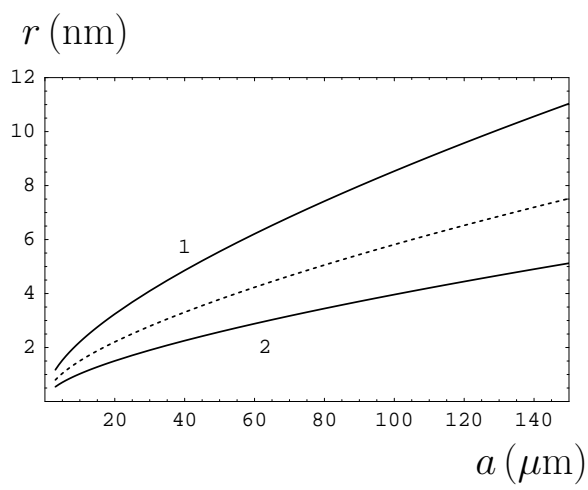

(b)

Figure 2. (a) Difference of the free energies of two hydrogen atoms located at $a=3 \mathrm{~nm}$ from the external and internal surfaces of carbon nanotube with fixed internal radius $R_{0}=10 \mathrm{~nm}$ (solid line) and fixed external radius $R=50 \mathrm{~nm}$ (dashed line) as a function of nanotube thickness. (b) Regions in the plane $(a, r)$ where the interatomic interaction of two hydrogen atoms is by at least 10 times smaller than their interaction to carbon nanotube with $R=50 \mathrm{~nm}, d=30 \mathrm{~nm}$ (region above line 1 ), by at least 10 times larger (region below line 2) and where both interactions are equal (the dashed line).

preferable. From this figure it is also obvious that for nanotubes of fixed thickness $d$ the value of $\mathcal{F}^{c, e x t}-\mathcal{F}^{c, \text { int }}$ is larger for the nanotubes of smaller external radius $R$. Thus, a multi-wall carbon nanotube tends to accumulate the hydrogen atoms in its interior.

In Fig. 2(b) we compare the van der Waals interaction energy between two hydrogen atoms separated by a distance $r$ to their interaction $\mathcal{F}^{c}$ with a multi-wall carbon nanotube. In the region of $(a, r)$-plane above line 1 the interatomic interaction is smaller than the interaction with a nanotube by at least a factor of 10 . In a similar manner, in the region below line 2 one can neglect by the interaction with a nanotube in comparison with the interatomic interaction. As to the region in between lines 1 and 2 , here both interactions are of the same order of magnitude and cannot be considered independently. The figures similar to Fig 2(b) permit to choose the conditions whereby the interatomic interactions can be neglected in comparison with the interaction of an atom with a nanotube.

The above results demonstrate that the multi-wall carbon nanotubes are of considerable promise for the problem of hydrogen storage and deserve further investigation.

\section{Acknowledgments}

This work was supported by the Russian Foundation for Basic Research (Grant No. 05-08-18119a). 


\section{References}

[1] Calbi M M, Cole M W, Gatica S M, Bojan M J and Stan G 2001 Rev. Mod. Phys. 73857

[2] Ding R G, Lu G Q, Yan Z F and Wilson M A 2001 J. Nanosci. Nanotech. 17

[3] Yildirim T and Ciraci S 2005 Phys. Rev. Lett. 94175501

[4] Bondarev I V and Lambin P 2005 Phys. Rev. B 72035451

[5] Dobson J F and Rubio A 2005 Preprint cond-mat/0502422

[6] Dag S, Ozturk Y, Ciraci S and Yildirim T 2005 Phys. Rev. B 72155404

[7] Yildirim T, Íñiguez J and Ciraci S 2005 Phys. Rev. B 72153403

[8] Turnbull J D and Boninsegni M 2005 Phys. Rev. B 71205421

[9] Blagov E V, Klimchitskaya G L and Mostepanenko V M 2005 Phys. Rev. B 71235401

[10] Lifshitz E M and Pitaevskii L P 1980 Statistical Physics, Part. II (Oxford: Pergamon Press)

[11] Milonni P W 1994 The Quantum Vacuum (San Diego: Academic Press)

[12] Mazzitelli F D 2004 Quantum Field Theory Under the Influence of External Conditions (Princeton: Rinton Press, Princeton) p 126

[13] Rauber S, Klein J R, Cole M W and Bruch L W 1982 Surf. Sci. 123173

[14] Johnson R E, Epstein S T and Meath W J 1967 J. Chem. Phys. 471271

[15] Handbook of Optical Constants of Solids, ed. Palik E D 1985 (New York: Academic Press)

[16] Mostepanenko V M and Trunov N N 1997 The Casimir Effect and its Applications (Oxford: Clarendon Press)

[17] Bordag M, Mohideen U and Mostepanenko V M 2001 Phys. Rep. 3531 